\title{
BELLS GALORE: \\ OSCILLATIONS AND CIRCLE-MAP DYNAMICS FROM SPACE-FILLING FRACTAL FUNCTIONS
}

\author{
Carlos E. Puente \\ Department of Land, Air and Water Resources \\ University of California, Davis, CA 95616 \\ cepuente@ucdavis.edu \\ Andrea Cortis \\ Earth Sciences Division \\ Lawrence Berkeley Laboratory, Berkeley, CA 94720 \\ acortis@lbl.gov \\ Bellie Sivakumar \\ Department of Land, Air and Water Resources \\ University of California, Davis, CA 95616 \\ sbellie@ucdavis.edu
}

\begin{abstract}
The construction of a host of interesting patterns over one and two dimensions, as transformations of multifractal measures via fractal interpolating functions related to simple affine mappings, is reviewed. It is illustrated that, while space-filling fractal functions most commonly yield limiting Gaussian distribution measures (bells), there are also situations (depending on the affine mappings' parameters) in which there is no limit. Specifically, the onedimensional case may result in oscillations between two bells, whereas the two-dimensional case may give rise to unexpected circle map dynamics of an arbitrary number of two-dimensional circular bells. It is also shown that, despite the multitude of bells over two dimensions, whose means dance making regular polygons or stars inscribed on a circle, the iteration of affine maps yields exotic kaleidoscopes that decompose such an oscillatory pattern in a way that is similar to the many cases that converge to a single bell.
\end{abstract}




\section{Introduction}

Recently, a multitude of interesting patterns (over one, two and three dimensions) defined as transformations of multifractal measures via fractal interpolating functions and the iterations of affine mappings have been uncovered..$^{1-4}$ Within this framework, it has also been established that Gaussian (normal distribution) patterns over one and two dimensions appear when the fractal functions fill up space, ${ }^{5-7}$ and that a wide variety of exotic kaleidoscopes decompose circular two-dimensional bells. ${ }^{8,9}$

The purpose of the present article is to show that, while the Gaussian limit is the most common case, there are also interesting cases (depending on the signs of some affine mapping parameters) in which there is no limiting convergence to a single Gaussian bell, but rather oscillations among a multitude of patterns that closely approximate bells. It is shown that whereas in the onedimensional case oscillations between two bells happen in one specific way, in the two-dimensional case oscillatory behavior among several two-dimensional circular bells may occur in two different ways that lead either to a roundabout motion or a star-shaped criss-crossed movement of an arbitrary number $n$ of bells, for $n>2$.

The organization of this paper is as follows. Given first is the mathematical construction of the interesting patterns over one dimension that may be constructed via the notion of projections and the iteration of affine mappings. Next, the conditions needed to define a limiting Gaussian pattern are explained followed by the specific scenario that would give rise to the limiting oscillation between two bells. Having established the results in one dimension, the article then studies generalizations to two-dimensional Gaussians to identify the relevant scenarios that define circle-map dynamics among a host of circular bells that jointly sketch beautiful kaleidoscopes of such everrotating attractors. The article ends with a summary and with some final remarks.

\section{Affine Mappings, Fractal Functions and Related Measures}

The graph $G$ of a fractal interpolating function, from $x$ to $y$ and passing by $N+1$ points on the plane $\left\{\left(x_{n}, y_{n}\right) ; x_{0}<\ldots<x_{N}, n=0,1, \ldots, N\right\}$, is 
defined as the unique attractor of $N$ affine maps as follows: ${ }^{10}$

$$
w_{n}\left(\begin{array}{l}
x \\
y
\end{array}\right)=\left(\begin{array}{cc}
a_{n} & 0 \\
c_{n} & d_{n}
\end{array}\right)\left(\begin{array}{l}
x \\
y
\end{array}\right)+\left(\begin{array}{c}
e_{n} \\
f_{n}
\end{array}\right), n=1, \ldots, N
$$

with the scaling parameters $d_{n}$ such that $\left|d_{n}\right|<1$, and with the other parameters, $a_{n}, c_{n}, e_{n}$, and $f_{n}$, satisfying the initial conditions:

$$
w_{n}\left(\begin{array}{l}
x_{0} \\
y_{0}
\end{array}\right)=\left(\begin{array}{l}
x_{n-1} \\
y_{n-1}
\end{array}\right), w_{n}\left(\begin{array}{l}
x_{N} \\
y_{N}
\end{array}\right)=\left(\begin{array}{l}
x_{n} \\
y_{n}
\end{array}\right), n=1, \ldots, N .
$$

Equations (2) ensure that the attractor $G$ exists and that it contains the initial interpolating points. Such conditions also allow computing the parameters $a_{n}, c_{n}, e_{n}$, and $f_{n}$ in terms of the scalings $d_{n}$ and the coordinates of the interpolating points, via simple linear equations. ${ }^{10}$ At the end, a unique, and hence deterministic, set $G$ is found that turns out to have a fractal dimension $D \in[1,2)$ that is given by the solution of: ${ }^{11}$

$$
\sum\left|d_{n}\right| a_{n}^{D-1}=1, \quad \text { if } \sum\left|d_{n}\right|>1
$$

or that equals 1 otherwise.

In a practical setting, the graph of a fractal function is obtained sampling the unique attractor point by point, starting the process at a point already in $G$ and progressively iterating the affine maps $w_{n}$ according to, for example, the outcomes of independent "coin" tosses. ${ }^{11}$ As this process is carried out, it happens that a unique invariant measure is also induced over $G$ that reflects how the attractor is being filled up. The existence of such a measure allows computing unique (and fully deterministic) projections over the coordinates $x$ and $y$ (say $d x$ and $d y$ ) that turn out to have irregular shapes as found in a variety of applications in geophysics and beyond. ${ }^{2-4}$

Figure 1 shows an example of these ideas for a fractal function that passes by the three points $\{(0,0),(1 / 2,-0.35),(1,-0.2)\}$, when the scalings of the two affine maps are $d_{1}=-0.8$ and $d_{2}=-0.6$. In addition to the graph of the attracting fractal function $f$, the figure includes the implied projections $d x$ and $d y$ of the unique measure over $G$ when the corresponding mappings $w_{1}$ and $w_{2}$ are iterated (15 million times) according to a 30-70\% proportion, using independent pseudo-random numbers, starting the process from the mid-point $(1 / 2,-0.35)$. 
As there is a lack of dependence of the coordinate $y$ on $x$, i.e. as implied by the zero value in the first component of the affine mappings (Equation (1)), the measure $d x$ is simply a deterministic binomial multifractal. ${ }^{12}$ The measure $d y$, in turn, being related to $d x$ via the deterministic self-affine fractal function, is just the derived measure of $d x$ via the function $f$ and is, hence, computed looking at all possible heights $y$ and adding the corresponding "probabilities" from "events" that emanate from $x .^{3}$

As is seen in Figure 1, the ideas lead to very interesting and "randomlooking" measures $d y$, which as in the above example resemble, for instance, a rainfall data set as a function of time. ${ }^{13}$ As multifractal measures have been found relevant in studies of turbulence, ${ }^{14}$, the projection sets given by these ideas, which turn out to perform a non-trivial fractional integration of a simple parent multifractal measure over $x$, may be assigned an interpretation as reflections or transformations of turbulence. ${ }^{15}$

\section{The Plane-Filling Cases and the Gaussian Limit}

When the interpolating points are equally spaced in $x$, all coefficients $a_{n}$ happen to be equal to $1 / N$ and hence the fractal dimension $D$ (Equation (3)) tends to 2 when the magnitudes of all the scalings $d_{n}$ 's tend to one. This implies, considering all possible sign combinations on such scalings, that there are $2^{N}$ routes toward obtaining plane-filling fractal interpolating functions.

Figure 2 shows an example of such limiting notions for a fractal function that passes by the three points $\{(0,0),(1 / 2,1),(1,0)\}$, when the scalings of the two required affine maps are $d_{1}=0.999$ and $d_{2}=-0.999$ (the "plus-minus" case) and when the two maps are iterated (50 million times) according to a 70-30\% proportion. As may be seen, a Gaussian distribution appears as a projection from a simple multifractal measure, indicating that the plane-filling fractal function has the ability to filter all the spikiness in $d x$ to produce, quite surprisingly, an ever-smooth $d y$.

This Gaussian limit turns out to be universal, as the same plane-filling fractal interpolating function gives a bell from an arbitrary iteration scheme. ${ }^{7}$ For the aforementioned three interpolating points, if the two mappings are iterated according to a $p-q$ proportion, with $q=1-p$, then the resulting bell simply has a mean $\mu$ equal to $p$. The result is yet more general as one 
may replace a simple binomial multifractal $d x$ by any measure defined over a continuum on $x$, to find yet another derived Gaussian limit. ${ }^{7}$

For the uniform measure in $x$, i.e. the $50-50 \%$ proportion with three equally-spaced points in $x$, some analytical results have been already established. ${ }^{7}$ In this scenario, the mean and variance are $\mu=1 / 2$ and $\sigma^{2}=$ $1 /\left(12-12 z^{2}\right)$, where $d_{1}=-d_{2}=z$ and $z$ tending to one. As the fractal function fills-up the plane when $z$ tends to one, i.e. as its range grows from $-\infty$ to $\infty$, the implied variance also goes to infinity. But, as shown in Table 1 , computed using the Maple V symbolic language package with a precision of 250 digits, the first twelve standardized moments of the derived measure over $y$, indeed converge, in an orderly manner, to the moments of the standard bell (as included in the last column). A formal proof of the Gaussian result for this case has already been presented and relies on showing (by induction) that all standardized moments of $d y$ converge to the moments of the standard Gaussian measure. ${ }^{7}$

The "minus-plus" case $\left(-d_{1}=d_{2}=z\right)$ turns out to yield a fractal interpolating function that is just the mirror image of the one found via the "plus-minus" case. As a consequence, when $z$ tends to one, a simple multifractal measure also yields a unique bell (but now with mean $q=1-p$ ) and the analytical results, just mentioned, also hold.

When $d_{1}=d_{2}=z$ (the "plus-plus" case) the fractal interpolating function becomes a symmetric "cloud" built by successive mid-point additions of powers of $z$ starting with the aforementioned three interpolating points. ${ }^{9}$ This case yields a derived measure eventually defined over the range $[0, \infty)$ whose mass progressively centers at infinity. For such a case, and for the 5050 proportion, one may compute analytically that, in the limit, the mean and the variance are $\mu=1 /(2-2 z)$ and $\sigma^{2}=1 /\left(12-12 z^{2}\right)$ (the same variance as before), which both tend to infinity as $z$ tends to one. This turns out to be a rather curious case, because the coefficient of variation (i.e. $\sigma / \mu$ ) tends to zero, indicating that in the limit the mass concentrates, with probability one, at infinity. As presented in Table 2, the lower order moments happen to behave in a manner (after standardization) that is progressively consistent with a standard Gaussian distribution, but a complete proof of the Gaussian limit is not available due to a lack of simplification on the required moment formulas. 


\section{Oscillations Between Two Bells in One Dimension}

Given the results presented in the previous section, consideration of the "minus-minus" case (i.e. $-d_{1}=-d_{2}=z$ ) would also be expected to give a Gaussian distribution in the limit when $z$ tends to one. For such a case, and for the interpolating points used before, the mean and the variance yield $\mu=1 / 4$ and $\sigma^{2}=1 /\left(12-12 z^{2}\right.$ ) (yet the same variance as before), and it appears that a single bell is found by considering the first twelve standardized moments as reported in Table 3. However, close examination of this case reveals that the limit does not contain just a single bell but rather oscillations between two bells, as follows.

When a graph similar to Figure 2 (based on 50 million iterations of suitable maps) is drawn for this case (not shown), the corresponding limiting bell-like measure exhibits noticeably larger oscillations around the mode than what is found for the "plus-minus" case. At first, this discrepancy appears to be a matter of a lack of precision in the calculations due to a small number of " 9 " s in say $z=0.999$, but further studies revealed that such was not the case.

If the mean from all points within the fractal interpolating function are computed, that is by following the binary tree of all successive (and equally spaced) additions up to a given level $n$, one encounters oscillatory behavior according to the formula:

$$
\mu_{n}=\frac{2^{n}+z(-2 z)^{n}}{\left(2^{n+1}+1\right)(z+1)} .
$$

Hence, in the limit, when $z$ tends to one, there happen to be two bells whose means oscillate between 0 and $1 / 2$, and therefore give an apparent average of $1 / 4$.

A similar study of the other sign combination cases (i.e. by layers) reveals convergence to a single mean, but the "minus-minus" case indeed yields unexpected oscillations, irrespective of the interpolating points used in the construction. As the non-standardized variance increases to infinity and as the difference in means remains finite for such a case, the resulting measure appears to be a single bell but this turns out not to be so, despite the information to the contrary implied by the first few moments in Table 3. 


\section{Extensions to Three Dimensions}

The expressions presented in Equations (1) and (2) may be extended to higher dimensions so that they produce attracting fractal functions living in three dimensions. Specifically, the graph $G$ of a fractal interpolating function, from $x$ into the plane $y-z$ and passing by $N+1$ points in three-dimensional space $\left\{\left(x_{n}, y_{n}, z_{n}\right) ; x_{0}<\ldots<x_{N}, n=0,1, \ldots, N\right\}$, is defined as the unique attractor of $N$ affine maps as follows: ${ }^{11}$

$$
w_{n}\left(\begin{array}{l}
x \\
y \\
z
\end{array}\right)=\left(\begin{array}{ccc}
a_{n} & 0 & 0 \\
c_{n} & d_{n} & h_{n} \\
k_{n} & l_{n} & m_{n}
\end{array}\right)\left(\begin{array}{c}
x \\
y \\
z
\end{array}\right)+\left(\begin{array}{c}
e_{n} \\
f_{n} \\
g_{n}
\end{array}\right), n=1, \ldots, N,
$$

such that

$$
A_{n}=\left(\begin{array}{cc}
d_{n} & h_{n} \\
l_{n} & m_{n}
\end{array}\right)=\left(\begin{array}{cc}
r_{n}^{(1)} \cos \theta_{n}^{(1)} & -r_{n}^{(2)} \sin \theta_{n}^{(2)} \\
r_{n}^{(1)} \sin \theta_{n}^{(1)} & r_{n}^{(2)} \cos \theta_{n}^{(2)}
\end{array}\right)
$$

has $L_{2}$-norm (i.e. the square root of the maximum eigenvalue of $A_{n}^{T} A_{n}$ ) less than 1 and subject to the initial conditions

$$
w_{n}\left(\begin{array}{l}
x_{0} \\
y_{0} \\
z_{0}
\end{array}\right)=\left(\begin{array}{l}
x_{n-1} \\
y_{n-1} \\
z_{n-1}
\end{array}\right), \quad w_{n}\left(\begin{array}{c}
x_{N} \\
y_{N} \\
z_{N}
\end{array}\right)=\left(\begin{array}{c}
x_{n} \\
y_{n} \\
z_{n}
\end{array}\right), n=1, \ldots, N .
$$

As these generalized fractal functions, with fractal dimensions now ranging from 1 to 3, are computed via iterations, such a process defines, once again, a unique measure over the new graph $G$ that may then be used to calculate a joint derived measure $d y z$ over the plane $y-z$ based on a simple multifractal measure $d x$ over $x$.

Figure 3 shows an example, consonant with Figure 1, of a three-dimensional fractal function that passes by the points $\{(0,0,0),(1 / 2,1,1),(1,0,0)\}$ and with parameters $A_{n}$ (Equation 6) given by,

$$
A_{1}=\left(\begin{array}{cc}
0 & 1 / 2 \\
1 / 2 & 0
\end{array}\right), \quad A_{2}=\left(\begin{array}{cc}
0 & -1 / 2 \\
1 / 2 & 0
\end{array}\right),
$$

that generates an interesting joint derived measure $d y z$ and non-trivial marginal measures $d y$ and $d z$, that is over the $y$ and $z$ directions. As before, these sets are obtained iterating the corresponding mappings $w_{1}$ and $w_{2}$ (Equation 5) 
based on 15 million iterations according to pseudo-random numbers, following a $70-30 \%$ proportion that also yields a simple binomial multifractal measure $d x$ over $x$.

As may be appreciated, these ideas lead to a host of interesting, complex and "random-looking" patterns $d y z$, which resemble sets found in geophysical applications such as rainfall-radar spatial patterns and contaminant plumes in ground waters. ${ }^{2,4,16}$ Surprisingly, the transformation of a turbulent-based multifractal measure via a "simple" three-dimensional fractal interpolating function encompasses the complex geometries of a class of natural sets and such a result hence suggests that the notions may one day be useful in devising a deterministic language for complexity. ${ }^{4,15}$

\section{Space-Filling Cases and the Two-Dimensional Gaussian Limit}

Increasingly space-filling fractal interpolating functions may be obtained as $\left\|A_{n}\right\|_{2} \rightarrow 1$ for all $n$. This leads, in polar coordinates (Equation 6), to the conditions $\left|r_{n}^{(j)}\right| \rightarrow 1, j=1,2 ; \theta_{n}^{(1)} \rightarrow \theta_{n}^{(2)}+k \pi$, for any integer $k{ }^{6}$ This yields $4^{N}$ possible paths towards space-filling functions when considering all possible sign combinations on the new scaling parameters $r_{n}^{(j)}$, as defined in Table 4 for $N=2$ affine mappings.

Figure 4 shows an example of such limiting notions for a fractal function that passes by the aforementioned three points $\{(0,0,0),(1 / 2,1,1),(1,0,0)\}$, when the parameter matrices $A_{n}$ take on the values,

$$
A_{1}=\left(\begin{array}{cc}
0 & 0.999 \\
0.999 & 0
\end{array}\right), \quad A_{2}=\left(\begin{array}{cc}
0 & -0.999 \\
0.999 & 0
\end{array}\right)
$$

and when the two maps are iterated (15 million times) according to a 70-30\% proportion. As may be seen, a circular joint Gaussian distribution appears as a projection from a simple multifractal measure, hence generalizing what was previously encountered with two-dimensional fractal functions.

Even though a complete proof of the Gaussianity of the bell shown in Figure 4 is not available yet as formulas defy simplification, it shall be shown that not all the sign combination cases in Table 4 result in unique bells over two dimensions. Leaving aside cases that show oscillations, as the "minus-minus" case in two dimensions, for a later section, the following are the trends that 
we have encountered for groups of sign combinations defined based on Table 4, and for the specific situation when $\theta_{1}=\theta_{1}^{(1)}=\theta_{1}^{(2)}$ and $\theta_{2}=\theta_{2}^{(1)}=\theta_{2}^{(2)}$.

Grouping the 16 cases on the scalings into 4 groups: $A=\operatorname{Cases}[1,7,10,16]$, $B=\operatorname{Cases}[2,4,13,15], C=\operatorname{Cases}[3,5,12,14]$, and $D=$ Cases $[6,8,9,11]$, it may be shown numerically (via suitable iterations) that the coefficient of correlation, $\rho$, of their corresponding bells are as follows,

Group $A: \rho=0$, except when $\theta_{1}=k \pi, \theta_{2}=l \pi$ that give $\rho=1$ or $\rho=-1$, for $k, l$ integers.

Group $B: \rho=0$, except when $\theta_{1}=k \pi$ that yields arbitrary correlation $\rho \in[-1,1]$, which depends on $\theta_{2}$ and the interpolating coordinates, for $k$ integer.

Group $C: \rho=0$, except when $\theta_{2}=l \pi$ that gives arbitrary correlation $\rho \in[-1,1]$, which depends on $\theta_{1}$ and the interpolating coordinates, for $l$ integer.

Group $D: \rho=0$, except when $\theta=\theta_{1}=\theta_{2}+k \pi$ that gives arbitrary correlation $\rho \in[-1,1]$, which depends on $\theta$ and the interpolating coordinates, for $k$ integer.

Further numerical calculation of means and variances, over both the $y$ and $z$ components, via iterations as well as by levels (as previously done on the two-dimensional case), reveals that the most common derived measures generated by the space-filling three-dimensional fractal functions are indeed single circular bells $(\rho=0)$ that have finite means, and variances that grow to infinity as the magnitude of the scalings tends to one.

There are, however, some noteworthy exceptions along the lines $\theta_{1}=k \pi$, $\theta_{2}=k \pi$ and $\theta_{1}=\theta_{2}+k \pi$, for an integer $k$, that generalize what was reported for the one-dimensional bell(s). The specific trends along such lines are as follows.

1. For $\theta=\theta_{1}=k \pi$ : (a) all cases in groups $A$ and $C$ and cases 8 and 9 (from group $D$ ) give a single circular bell having finite means; (b) cases 2 and 6 (from groups $B$ and $D$, respectively) yield a single bell whose mean converges to $(\infty, \infty)$, while cases 4 and 11 (also from groups $B$ and $D$ ) result in another single bell whose mean converges to $(\infty,-\infty)$; and (c) cases 13 and 15 (from group $B$ ), which generate non-circular bells as a function of $\theta$, give two oscillating bells, as in the one-dimensional case. 
2. For $\theta=\theta_{2}=k \pi$ : (a) all cases in groups $A$ and $B$ and cases 8 and 9 give a single circular bell with finite means; (b) cases 3 and 6 result in a single bell centered at $(\infty, \infty)$, whereas cases 5 and 11 yield bells with means $(-\infty, \infty)$; and (c) cases 12 and 14 result in two oscillating elliptical bells.

3. For $\theta=\theta_{1}=\theta_{2}+k \pi$ : (a) all cases in groups $B$ and $C$ and cases 7 and 10 give a single circular bell with finite means; (b) cases 6 and 11 yield single bells whose mean tends respectively to $(\infty, \infty)$ and $(\infty,-\infty) ;($ c) cases 8 and 9 , yielding non-zero correlation, correspond (contrary to the results just reported for the other $\theta$ lines) to single bells with finite means; and (d) cases 1 and 16 appear to converge to a single bell with finite means, but close examination of the results reveal that they contain oscillatory behavior that encompasses $2 \pi / \theta$ bells, as explained in the next section.

\section{Multiple Bells and Their Circle Map Dynamics}

When all scalings $r_{n}^{(j)}$ are positive (case 1) or negative (case 16), and when the angles $\theta_{1}=\theta_{1}^{(1)}=\theta_{1}^{(2)}$ and $\theta_{2}=\theta_{2}^{(1)}=\theta_{2}^{(2)}$ are such that $\theta=\theta_{1}=\theta_{2}+k \pi$, for $k$ integer, one encounters seemingly only a single circular bell, but detailed calculations by levels indicate that there are oscillations among a host of bells depending on the angle $\theta$.

As illustrated in Figure 5 for the "all plus" case with angles $\theta$ equal to $2 \pi$ over $3,4,5,6,7$, and 8, the bell's center travels following a circle, centered at the apparent single mean, and in consonance with the simple circle map $\phi_{n+1}=\phi_{n}+\theta$. As seen, the radius of such a circle increases as $\theta$ decreases, for the line joining the points $(0,0)$ and $(1 / 2,1 / 2)$ is always inscribed within such a circle. When $2 \pi / \theta=n$, the circle map generates a regular polygon having $n$ sides and hence such a case corresponds to $n$ bells that cycle (counterclockwise) in such a circle. When $\theta$ does not divide $2 \pi$, case 1 generates infinitely many bells whose means travel in a circle.

Figure 6 shows what happens level by level in the "all minus" case. As may be inferred, such a sign combination case also yields oscillations among several bells, now consonant with the simple dynamics on a circle, $\phi_{n+1}=\phi_{n}+\pi-\theta$, which result sometimes in simple polygons (for large angles $\theta$ ) but most often stars inscribed on a finite circle that contains, once again, the line joining the 
points $(0,0)$ and $(1 / 2,1 / 2)$. When $2 \pi / \theta=n$ and $n>6$ all patterns are stars and the simple map travels within the circle (clockwise) yielding sharp oscillatory behavior on the means of the implied $n$ bells. As before, this case yields infinitely many bells when $\theta$ does not divide $2 \pi$.

It should be stressed that cases 1 and 16 only give many bells along the line $\theta=\theta_{1}=\theta_{2}+k \pi$, for outside such the dynamics no longer happen along a circle but rather are attracted towards a true center yielding, at the end, a single bell with finite mean, as reported earlier.

\section{Exotic Decompositions of Oscillatory Bells}

As previously reported, ${ }^{8,9}$ when the aforementioned angles associated with the two affine mappings $\theta_{1}$ and $\theta_{2}$ both divide $2 \pi$ and when such are multiples of one another, exotic kaleidoscopes of patterns end up decomposing single bells in non-trivial manners. ${ }^{9}$ It happens that such a behavior turns out to be valid not only for the cases that define single bells with finite means but also for the two oscillatory cases explained in the previous section.

As an illustration, Figure 7 shows examples of the beautiful transient patterns that decompose the oscillatory attractors for the two cases, when the binary expansion of $\pi$ is used to guide the iterations and when the scalings have magnitudes equal to $1-\epsilon$ with $\epsilon=10^{-8}$. Remarkably, despite the cyclic movement of the dancing bells, the patterns obtained do not exhibit within them oscillations of any kind and (as in cases defining single bells) their successive superposition gives an attractor that appears to define a single bell. ${ }^{9}$

\section{Summary}

This work has illustrated that the transformation of simple multifractal measures, via fractal interpolating functions (defined over two and three dimensions and computed through the iteration of simple affine mappings), gives rise to: (a) interesting and seemingly-random complex patterns if the dimension of the fractal function is "low," and (b) limiting Gaussian or "closely" Gaussian-like measures when the fractal functions fill up the space in which they live. 
It has been explained how the nature of the limiting Gaussian measures (the bells) turns out to depend on the sign combinations of the scaling parameters of the affine mappings, as follows. For the two-dimensional fractal functions via the use of two mappings, there are four cases. Two of them, the "plus-minus" and "minus-plus" cases, may indeed be proven to define a Gaussian limit that prior to standardization has a finite mean. There is a case, the "plus-plus" one, that appears also to converge to a Gaussian, but such is substantially different as it has a mean that converges to infinity (prior to standardization) and a coefficient of variation that tends to zero. The remaining "minus-minus" case turns out to be surprising, for calculation of all points within the fractal function, by levels, shows convergence to two oscillating bells (with finite alternating means before standardization) rather than just one.

For the three-dimensional fractal functions and via two affine mappings, there are sixteen different sign combination cases. Whereas the most common behavior obtained turns out to be convergence to a single circular Gaussian distribution with finite means, there are also cases that mimic the results obtained with the two-dimensional fractal functions. For instance, and similar to the "plus-plus" case, there are cases that appear to lead to single bells that drift to the corners of the four quadrants, and there are also sign combinations that, as the "minus-minus" case, result in oscillations among several bells. In regards to the latter, it has been shown, analyzing the process by levels, that the "all plus" and "all minus" cases surprisingly lead to oscillations among an arbitrary number of two-dimensional bells that follow circle-map dynamics and that result in lovely transient kaleidoscopic patterns that decompose the limiting attractors.

Acknowledgments This work was supported in part by the Director, Office of Science, of the U.S. Department of Energy under Contract No. DE-AC02$05 \mathrm{CH} 11231$. 


\section{References}

1. C. E. Puente, "Multinomial Multifractals, Fractal Interpolators, and the Gaussian Distribution," Phys. Lett. A 161, 441-447 (1992).

2. C. E. Puente, "A Fractal-Multifractal Approach to Geostatistics," in Geostatistics for the Next Century, ed. R. Dimitrakopoulos (Kluwer Academic Publishers, Dordrecht, 1994) pp. 476-487.

3. C. E. Puente, "A New Approach to Hydrologic Modeling: Derived Distributions Revisited," J. Hydrol. 187, 65-80 (1996).

4. C. E. Puente, "A Universe of Projections: May Plato be Right?," Chaos, Solitons $\& 3$ Fractals 19, 241-253 (2004).

5. C. E. Puente, "Deterministic Fractal Geometry and Probability," Int. J. Bifurc. Chaos 4(6), 1613-1629 (1994).

6. C. E. Puente and A. Klebanoff, "Gaussians Everywhere," Fractals, 2(1), 65-79 (1994).

7. C. E. Puente, M. M. López, J. E. Pinzón and J. M. Angulo, "The Gaussian Distribution Revisited," Adv. Appl. Prob., 28(2), 500-524 (1996).

8. C. E. Puente, "The Remarkable Kaleidoscopic Decompositions of the Bivariate Gaussian Distribution," Fractals, 5(1), 47-61 (1997).

9. C. E. Puente, Treasures Inside the Bell. Hidden Order in Chance (World Scientific, Singapore, 2003).

10. M. F. Barnsley, "Fractal Functions and Interpolation," Constr. Approx. 2, 303-329 (1986).

11. M. F. Barnsley, Fractals Everywhere (Academic Press, New York, 1988).

12. B. B. Mandelbrot, "Multifractal Measures Especially dor the Geophysicist," in Fractals in Geophysics, eds. C. H. Scholz and B. B. Mandelbrot (Birkhauser Verlag, Basel, 1989) pp. 1-42.

13. C. E. Puente and N. Obregón, "A Deterministic Geometric Representation of Temporal Rainfall. Results for a Storm in Boston," Water Resour. Res. 32(9), 2825-2839 (1996). 
14. C. Meneveau and K. R. Sreenivasan, "Simple Multifractal Cascade Model for Fully Developed Turbulence," Phys. Rev. Lett. 59, 14241427 (1987).

15. C. E. Puente and B. Sivakumar, "Modeling Hydrologic Complexity: A Case for Geometric Determinism," Hydrol. Earth Syst. Sci. 11, 721-724 (2007).

16. C. E. Puente, O. Robayo, M. C. Díaz and B. Sivakumar, "A fractalmultifractal approach to groundwater contamination. 1. Modeling conservative tracers at the Borden site," Stoch. Environ. Res. Risk Assess. 15(5), 357-371 (2001). 


\section{List of Figures}

1. From a multifractal measure $d x$ to a derived measure $d y$ via a fractal interpolating function $f$. (The scale in $x$ is from 0 to 1 , and the one in $y$ is from -0.38 to 0.06 . The vertical scales in $d x$ and $d y$ are not given but both measures are normalized so that they add up to one).

2. From a multifractal measure $d x$ to a derived Gaussian measure $d y$ via a space-filling fractal interpolating function $f$.

3. From a multifractal measure $d x$ to a joint derived measure $d y z$ (with marginals $d y$ and $d z$ ) via a fractal interpolating function $f$ from $x$ to $y-z$, shown in the $x-y$ and $x-z$ planes.

4. From a multifractal measure $d x$ to a derived joint circular Gaussian measure $d y z$ via a space-filling fractal interpolating function $f$.

5. Oscillations on bell's means computed by layers for "all positive" scalings case. The angle $\theta$ takes on the values $2 \pi / 3,2 \pi / 4,2 \pi / 5,2 \pi / 6,2 \pi / 7$, and $2 \pi / 8$ (shown left to right and top to bottom).

6. Oscillations on bell's means computed by layers for "all negative" scalings case. The angle $\theta$ takes on the values $2 \pi / 3,2 \pi / 4,2 \pi / 5,2 \pi / 6,2 \pi / 7$, and $2 \pi / 8$ (shown left to right and top to bottom).

7. Selected exotic kaleidoscopic patterns inside oscillating bells. "All positive" scalings case (left) with parameters $\{0,0,0),(1 / 2,1,1),(1,0,0)\}$, $\theta_{1}=2 \pi / 11, \theta_{2}=6 \pi / 11$ and "all negative" scalings case (right) with parameters $\{0,0,0),(1 / 2,1,-3),(1,5,0)\}, \theta_{1}=2 \pi / 5, \theta_{2}=\pi / 5$. Shown patterns are made up of 20,000 dots. 
Table 1. Standardized central moments of $d y$ for the plus-minus case. Parameters: $d_{1}=-d_{2}=z$ and $\{(0,0),(1 / 2,1),(1,0)\}$.

\begin{tabular}{|c||c|c|c||c|}
\hline Order & $z=0.9999$ & $z=0.999999$ & $z=0.99999999$ & $\mathcal{N}(0,1)$ \\
\hline 3 & 0.000000000 & 0.000000000 & 0.000000000 & 0.0 \\
4 & 2.999365046 & 2.999993650 & 2.999999937 & 3.0 \\
5 & 0.000000000 & 0.000000000 & 0.000000000 & 0.0 \\
6 & 14.990478199 & 14.999904750 & 14.999999048 & 15.0 \\
7 & 0.000000000 & 0.000000000 & 0.000000000 & 0.0 \\
8 & 104.866744091 & 104.998666509 & 104.999986665 & 105.0 \\
9 & 0.000000000 & 0.000000000 & 0.000000000 & 0.0 \\
10 & 943.002111830 & 944.979997736 & 944.999799975 & 945.0 \\
11 & 0.000000000 & 0.000000000 & 0.000000000 & 0.0 \\
12 & 10362.054001516 & 10394.669964570 & 10394.996699588 & 10395.0 \\
\hline
\end{tabular}

Table 2. Standardized central moments of $d y$ for the plus-plus case. Parameters: $d_{1}=d_{2}=z$ and $\{(0,0),(1 / 2,1),(1,0)\}$.

\begin{tabular}{|c||c|c|c||c|}
\hline Order & $z=0.9999$ & $z=0.999999$ & $z=0.99999999$ & $\mathcal{N}(0,1)$ \\
\hline 3 & -0.032656325 & -0.003265983 & -0.000326599 & 0.0 \\
4 & 3.001639399 & 3.000016400 & 3.000000164 & 3.0 \\
5 & -0.326658273 & -0.032659923 & -0.003265986 & 0.0 \\
6 & 15.035259628 & 15.000352666 & 15.000003527 & 15.0 \\
7 & -3.432783098 & -0.342932064 & -0.034292860 & 0.0 \\
8 & 105.643263393 & 105.006430686 & 105.000064307 & 105.0 \\
9 & -41.260116637 & -4.115251502 & -0.411514386 & 0.0 \\
10 & 956.901068056 & 945.118861509 & 945.001188600 & 945.0 \\
11 & -568.823119975 & -56.586203271 & -5.658324296 & 0.0 \\
12 & 10628.732737598 & 10397.330855430 & 10395.023307907 & 10395.0 \\
\hline
\end{tabular}


Table 3. Standardized central moments of $d y$ for the minus-minus case. Parameters: $-d_{1}=-d_{2}=z$ and $\{(0,0),(1 / 2,1),(1,0)\}$.

\begin{tabular}{|c||c|c|c||c|}
\hline Order & $z=0.9999$ & $z=0.999999$ & $z=0.99999999$ & $\mathcal{N}(0,1)$ \\
\hline 3 & 0.000002939 & 0.000000003 & 0.000000000 & 0.0 \\
4 & 2.999080244 & 2.999990800 & 2.999999908 & 3.0 \\
5 & 0.000029380 & 0.000000029 & 0.000000000 & 0.0 \\
6 & 14.986209090 & 14.999862001 & 14.999998620 & 15.0 \\
7 & 0.000308244 & 0.000000309 & 0.000000000 & 0.0 \\
8 & 104.807032780 & 104.998068023 & 104.999980680 & 105.0 \\
9 & 0.003694899 & 0.000003704 & 0.000000004 & 0.0 \\
10 & 942.107516384 & 944.971020552 & 944.999710200 & 945.0 \\
11 & 0.050733888 & 0.000050923 & 0.000000051 & 0.0 \\
12 & 10347.314696658 & 10394.521843197 & 10394.995218301 & 10395.0 \\
\hline
\end{tabular}

Table 4. Sign combinations on scaling parameters for three-dimensional fractal interpolating functions based on two affine mappings.

\begin{tabular}{|ccccc||ccccc|}
\hline Case & $r_{1}^{(1)}$ & $r_{1}^{(2)}$ & $r_{2}^{(1)}$ & $r_{2}^{(2)}$ & Case & $r_{1}^{(1)}$ & $r_{1}^{(2)}$ & $r_{2}^{(1)}$ & $r_{2}^{(2)}$ \\
\hline 1 & + & + & + & + & 9 & - & + & + & - \\
2 & + & + & + & - & 10 & - & - & + & + \\
3 & + & - & + & + & 11 & - & + & - & + \\
4 & + & + & - & + & 12 & + & - & - & - \\
5 & - & + & + & + & 13 & - & - & + & - \\
6 & + & - & + & - & 14 & - & + & - & - \\
7 & + & + & - & - & 15 & - & - & - & + \\
8 & + & - & - & + & 16 & - & - & - & - \\
\hline
\end{tabular}



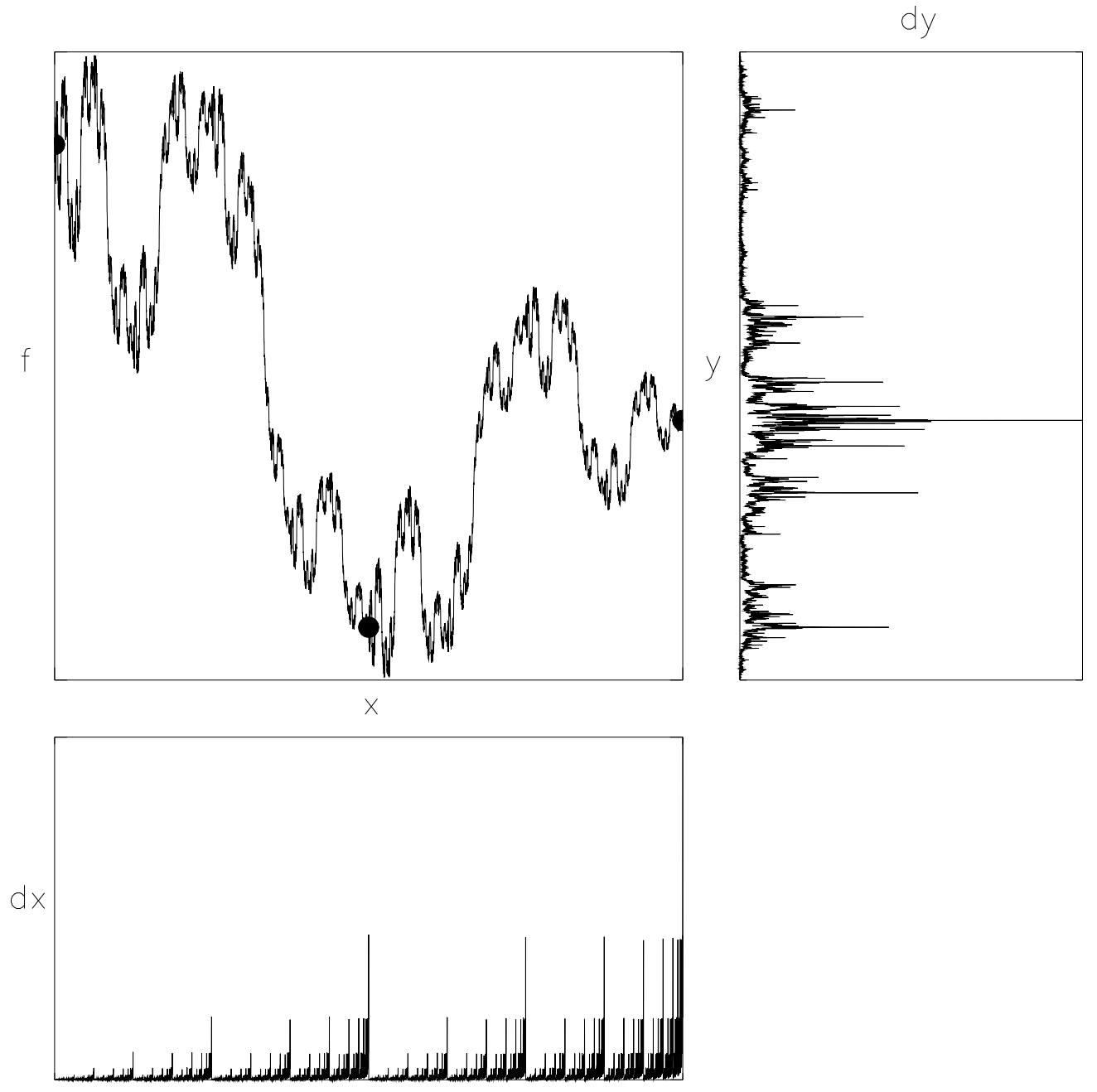

Figure 1 


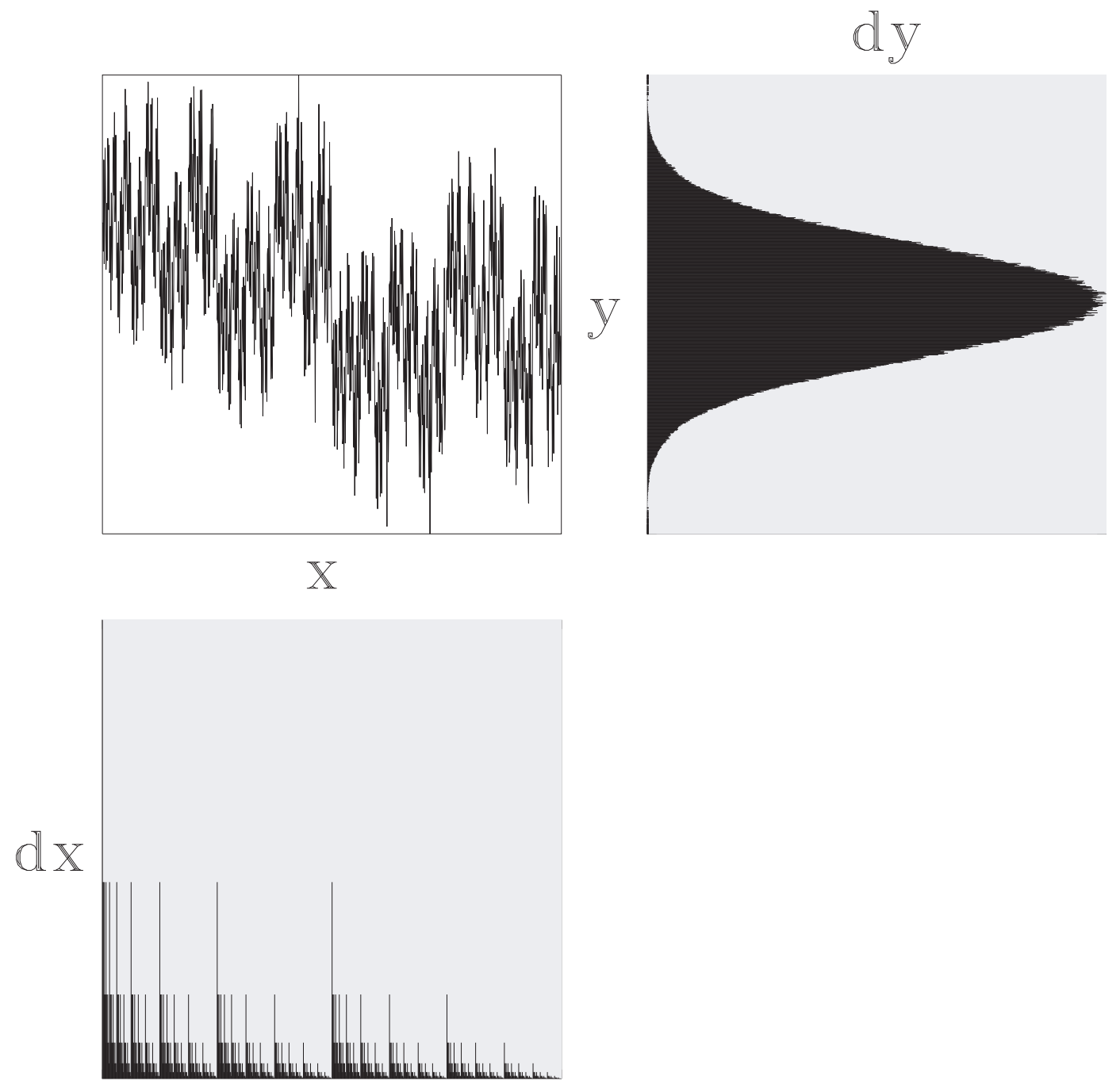

Figure 2 


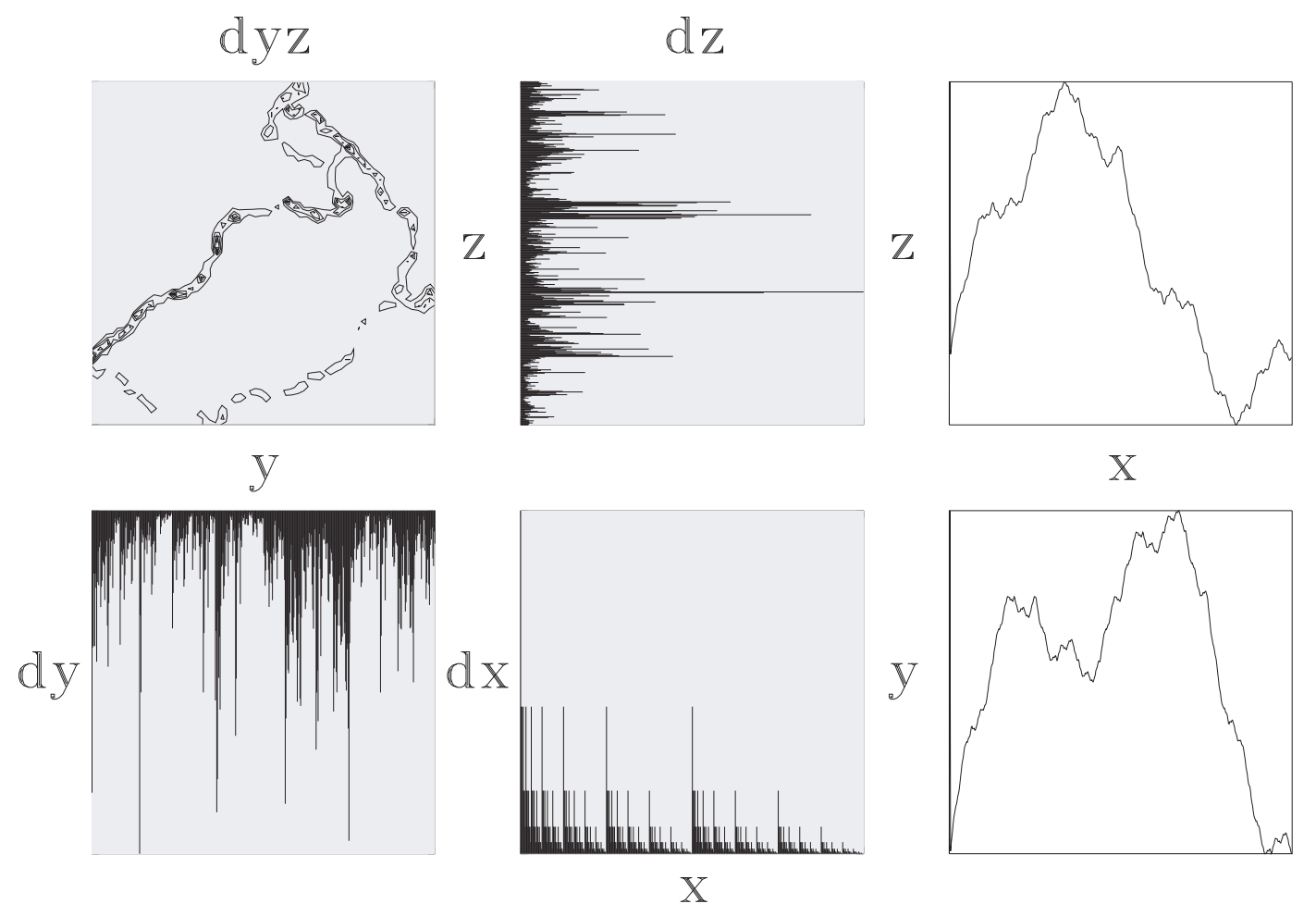

Figure 3 


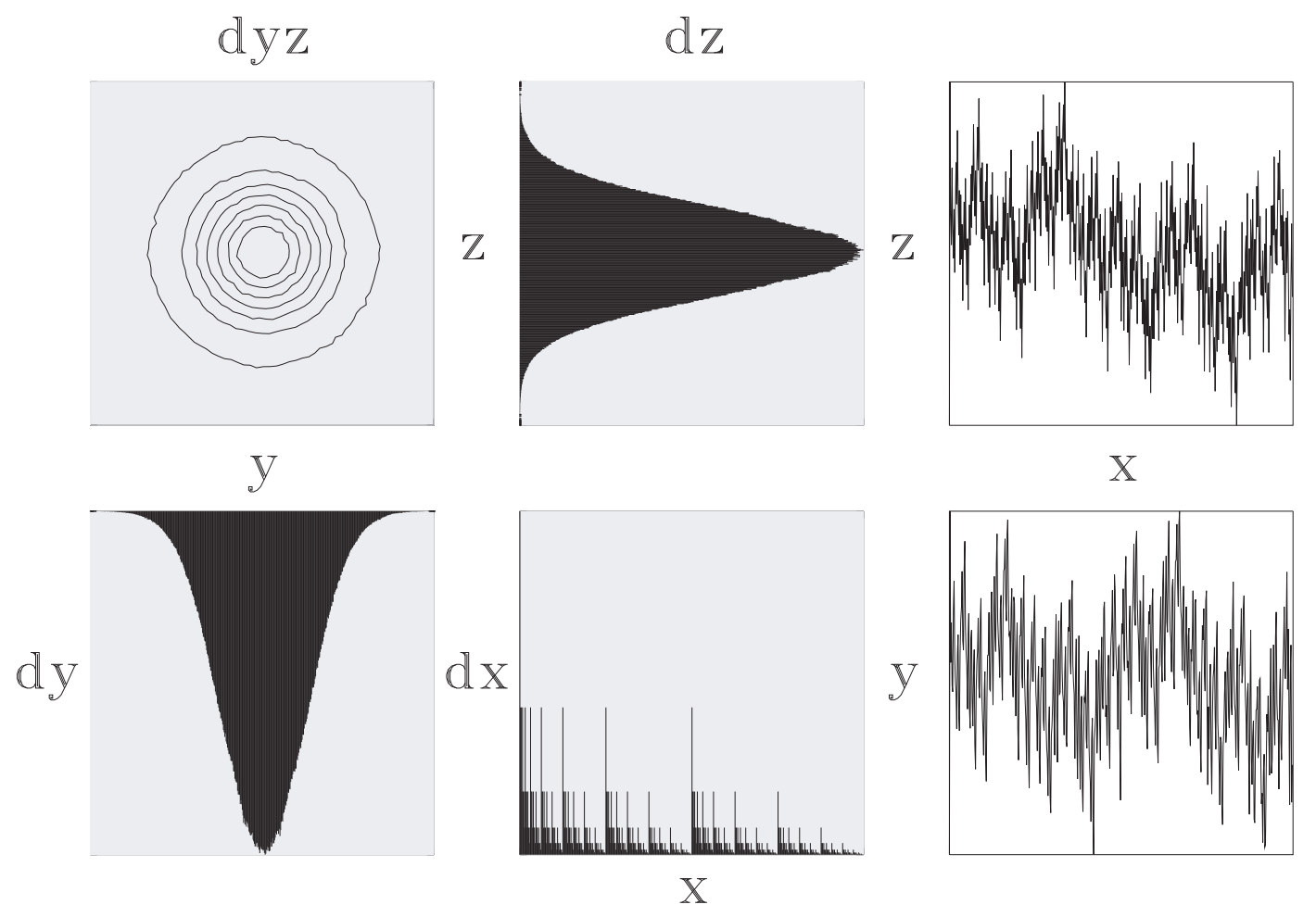

Figure 4 

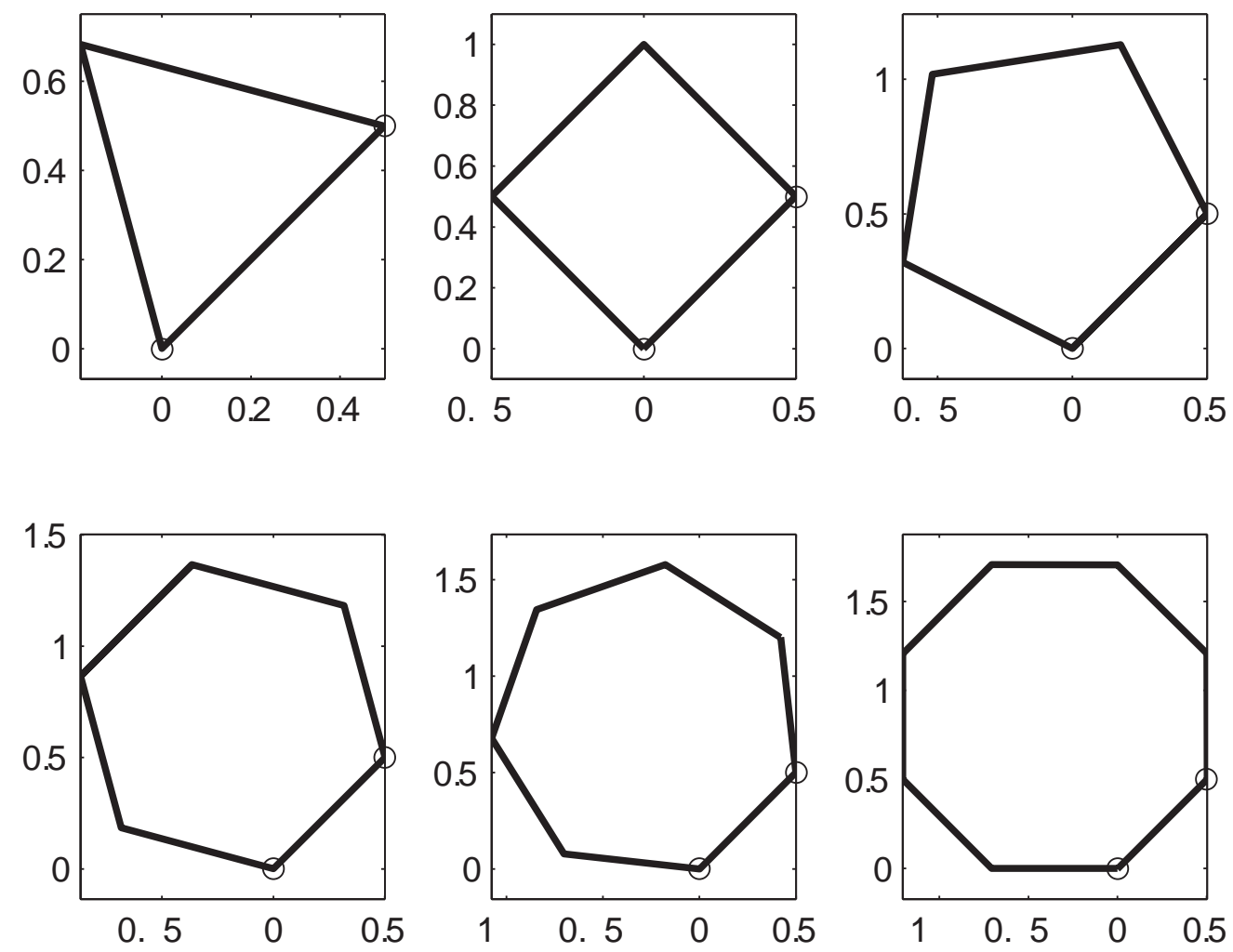

Figure 5 

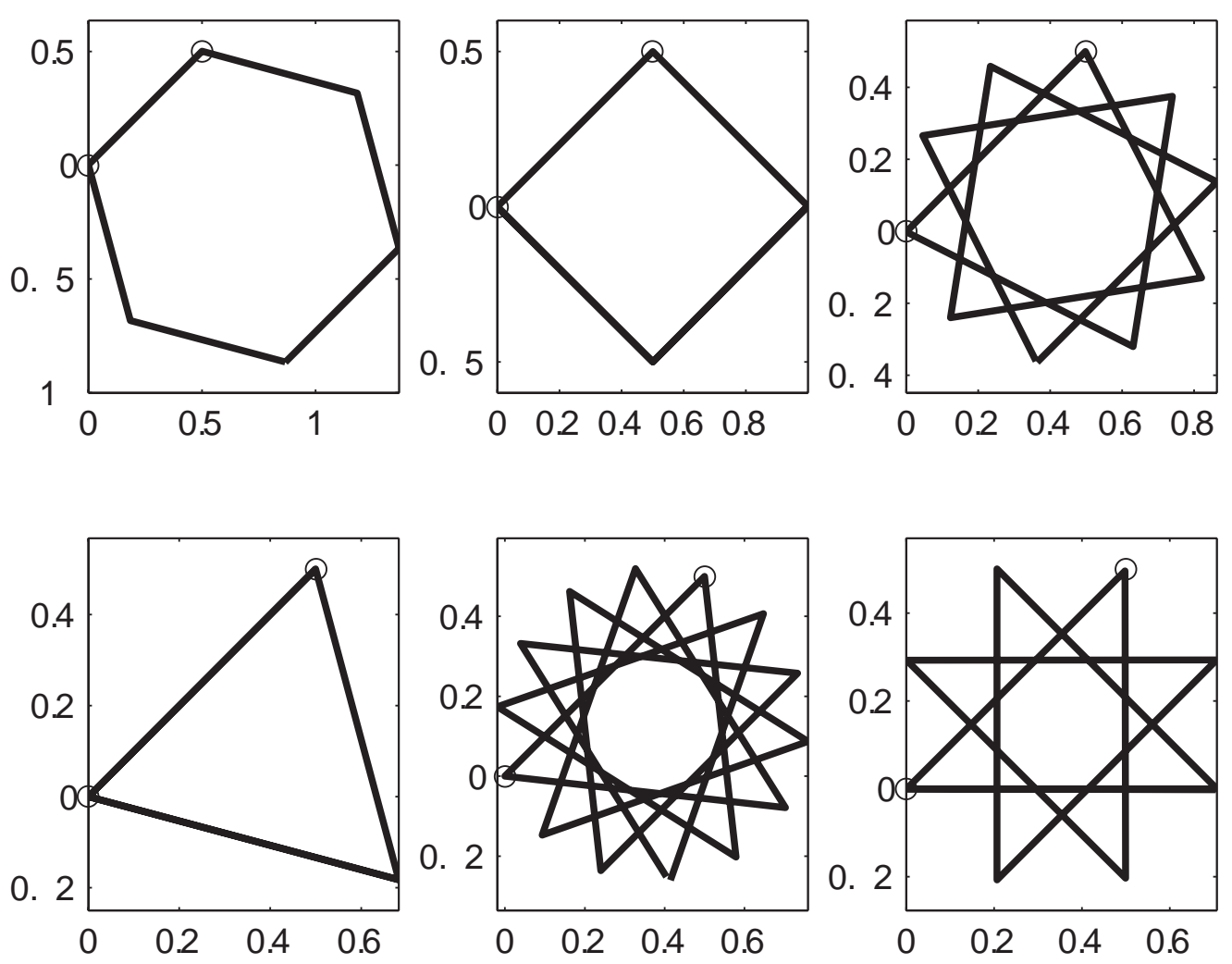

Figure 6 

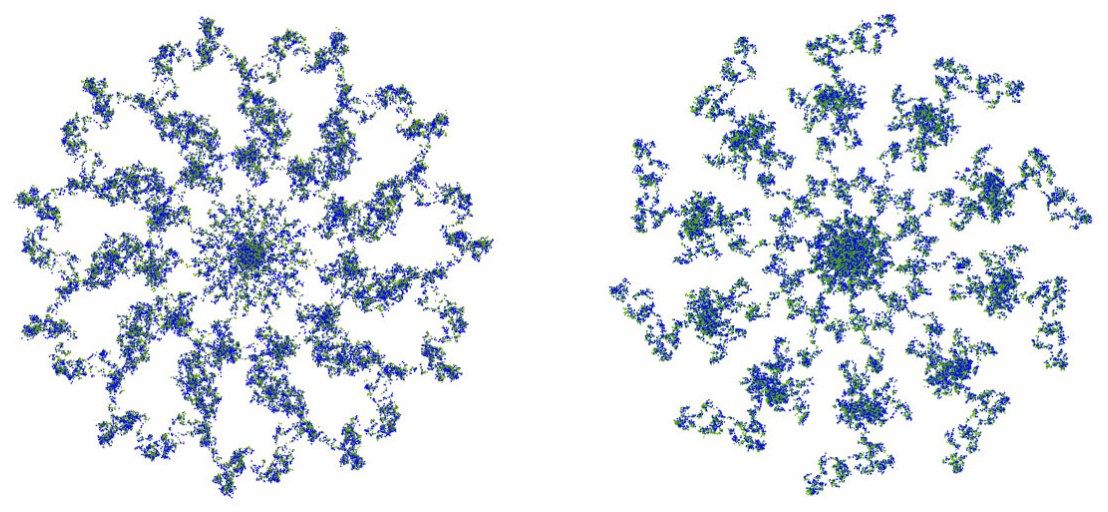

Figure 7 